\title{
An Exact Solution for a Boundary Value Problem with Application in Fluid Mechanics and Comparison with the Regular Perturbation Solution
}

\author{
Abdelhalim Ebaid ${ }^{1}$ and S. M. Khaled ${ }^{2,3}$ \\ ${ }^{1}$ Department of Mathematics, Faculty of Science, University of Tabuk, P.O. Box 741, Tabuk 71491, Saudi Arabia \\ ${ }^{2}$ Department of Mathematics, Faculty of Sciences, Helwan University, Cairo, Egypt \\ ${ }^{3}$ Department of Studies and Basic Sciences, Faculty of Community, University of Tabuk, Saudi Arabia
}

Correspondence should be addressed to Abdelhalim Ebaid; aebaid@ut.edu.sa

Received 29 January 2014; Accepted 2 March 2014; Published 6 April 2014

Academic Editor: Robert A. Van Gorder

Copyright (C) 2014 A. Ebaid and S. M. Khaled. This is an open access article distributed under the Creative Commons Attribution License, which permits unrestricted use, distribution, and reproduction in any medium, provided the original work is properly cited.

\begin{abstract}
The exact solution for any physical model is of great importance in the applied science. Such exact solution leads to the correct physical interpretation and it is also useful in validating the approximate analytical or numerical methods. The exact solution for the peristaltic transport of a Jeffrey fluid with variable viscosity through a porous medium in an asymmetric channel has been achieved. The main advantage of such exact solution is the avoidance of any kind of restrictions on the viscosity parameter $\alpha$, unlike the previous study in which the restriction $\alpha \ll 1$ has been put to achieve the requirements of the regular perturbation method. Hence, various plots have been introduced for the exact effects of the viscosity parameter, Daray's number, porosity, amplitude ratio, Jeffrey fluid parameter, and the amplitudes of the waves on the pressure rise and the axial velocity. These exact effects have been discussed and further compared with those approximately obtained in the literature by using the regular perturbation method. The comparisons reveal that remarkable differences have been detected between the current exact results and those approximately obtained in the literature for the axial velocity profile and the pressure rise.
\end{abstract}

\section{Introduction}

The subject of peristaltic flow, first introduced and formulated by Latham [1], has been a field of very active research during the last few decades due to its important applications in many scientific fields such as engineering, medicine, and biology. Such applications for peristaltic flow appear in the transport of bile in the bile duct, vasomotion of the small blood vessels, the transport of urine from kidney to the bladder, the movement of eggs in the fallopian tube, the transport of the spermatozoa in cervical canal, the chyme movement in the intestine, and the transport of intrauterine fluid within the cavity of the uterus. In addition, the mechanism of peristaltic transport has been exploited for industrial applications like sanitary fluid transport, transport of corrosive fluids where the contact of the fluid with the machinery parts is prohibited, and transport of a toxic liquid which is used in nuclear industry to avoid contamination from the outside environment. Since Latham [1], many authors investigated too many problems for the peristaltic flow of Newtonian and nonNewtonian fluids under different boundary conditions. In this regard, several models have been investigated by Shapiro et al. [2], Zien and Ostrach [3], Lee and Fung [4], Srivastava et al. [5], Takabatake et al. [6], L. M. Srivastava and V. P. Srivastava [7], Tang and Shen [8], Misra and Pandey [9, 10], V. P. Srivastava and L. M. Srivastava [11], Eytan et al. [12], Vajravelu et al. [13], and Mekheimer and Abd elmaboud [14, 15] to describe peristaltic flow in symmetric and asymmetric channels or axisymmetric tubs.

Recently, the peristaltic flow in asymmetric channel has attracted much attention due to the physiological observations that the intrauterine fluid flow induced by myometrial contractions is peristaltic-type motion. These contractions occur in asymmetric directions during the secretory phase, 
when the embryo enters the uterus for implantation, de Vries et al. [16]. The main notice on these aforementioned studies is that the fluid viscosity is assumed to be constant. However, such assumption is not valid everywhere, where the coefficients of viscosity for real fluids are functions of space coordinate, temperature, and pressure. For many liquids, such as water, oils, and blood, the variation of viscosity due to space coordinate and temperature change is more dominant than other effects. Accordingly, El Hakeem et al. [17] studied the hydromagnetic peristaltic flow of fluid with variable viscosity in a uniform tube under zero Reynolds number with long wavelength approximation. They considered that the viscosity of the fluid varies across the thickness of the duct. Later, many authors [18-22] have analyzed the peristaltic transport of various types of fluids taking into account the variation of viscosity. Very recently, Afsar Khan et al. [22] discussed the peristaltic flow of a Jeffrey fluid with variable viscosity through a porous medium in an asymmetric channel.

In such kind of problems, the authors usually expand the viscosity function in terms of a small viscosity parameter and hence consider the first two or three terms of Maclaurin series. This procedure helped them to use the regular perturbation method to solve the differential equations governing the flow. Consequently, the perturbation series solutions up to first order [17] or second order [22] were obtained in terms of such small viscosity parameter. These approximate solutions have been used to derive some numerical results for the effects of various physical parameters on the fluid velocity and the pressure gradient. In this regard, we believe that such approximate solutions do not always lead to the correct physical interpretations, especially when the issue of convergence is not addressed. Moreover, when we use the regular perturbation method as a method of solution we do not know the order that we can stop at to achieve numerical results with good accuracy. In general, the accuracy of the approximate solutions derived from any approximate analytical method cannot be checked without addressing the issue of convergence. When it is difficult to study such convergence, one other way to check accuracy, especially in the absence of the exact solutions, is to compare the obtained results with highly trust numerical methods that applied to solve the same problems. In order to indicate our point of view we will reinvestigate the problem solved very recently by Afsar Khan et al. [22] by using the regular perturbation method. Therefore, the objective of the present study is to confirm our belief that the approximate solutions deduced from the regular perturbation method do not always lead to correct physical solutions. This goal will be achieved via the following three steps:

(1) obtaining the exact solution for the differential equation governing the axial velocity;

(2) obtaining the exact expressions for the pressure gradient and the pressure rise;

(3) comparing the present exact results for the fluid velocity and the pressure rise with those approximately obtained by using the regular perturbation method [22], at the same values of physical parameters.

\section{The Physical Problem}

Afsar Khan et al. [22] considered the peristaltic flow of an incompressible Jeffrey fluid in an asymmetric channel of width $a_{1}+a_{2}$. Sinusoidal wave propagating with constant speed $c$ on the channel walls induces the flow. The wall surfaces are chosen in the following forms:

$$
\begin{array}{cc}
H_{1}(X, t)=a_{1}+b_{1} \cos \left[\frac{2 \pi}{\lambda}(X-c t)\right], & \text { Upper wall, } \\
H_{2}(X, t)=-a_{2}-b_{2} \cos \left[\frac{2 \pi}{\lambda}(X-c t)+\phi\right], & \text { Lower wall, }
\end{array}
$$

where $b_{1}, b_{2}$ are amplitude of the upper and lower waves, $\lambda$ is the wavelength, and $\phi$ is the phase difference which varies in the range $0 \leq \phi \leq \pi$. In addition, $a_{1}, a_{2}, b_{1}, b_{2}$, and $\phi$ should satisfy the following condition [22]:

$$
b_{1}^{2}+b_{2}^{2}+2 b_{1} b_{2} \cos \phi \leq\left(a_{1}+a_{2}\right)^{2} .
$$

The flow is assumed to be steady in the wave frame $(x, y)$ moving with velocity $c$ away from the fixed frame $(X, Y)$. The transformation between these two frames is given by

$$
\begin{gathered}
x=X-c t, \quad y=Y, \quad u=U-c, \\
p(x)=P(X, t),
\end{gathered}
$$

where $u$ and $v$ are the velocity components in the wave frame $(x, y)$ and $p$ and $P$ are pressure in wave and fixed frame of reference, respectively. Afsar Khan et al. [22] found that under the assumptions of long wavelength and low Reynolds number approximation the flow is governed by the following system of partial differential equations in nondimensional form:

$$
\begin{gathered}
\frac{\partial p}{\partial y}=0 \\
\frac{\partial p}{\partial x}=\frac{1}{\epsilon} \frac{\partial}{\partial y}\left[\frac{\mu(y)}{\left(1+\lambda_{1}\right)} \frac{\partial u}{\partial y}\right]-\frac{\mu(y)}{\mathrm{Da}}(u+1)
\end{gathered}
$$

where $\epsilon$ is the porosity of the porous medium, $\lambda_{1}$ is the ratio of relaxation to retardation times, and $\mathrm{Da}$ is Darcy's number. Equation (4) shows that $p$ is independent of $y$. Accordingly, (5) can be written as

$$
\frac{d p}{d x}=\frac{1}{\epsilon} \frac{\partial}{\partial y}\left[\frac{\mu(y)}{\left(1+\lambda_{1}\right)} \frac{\partial u}{\partial y}\right]-\frac{\mu(y)}{\mathrm{Da}}(u+1) .
$$

The flow is governed by the following boundary conditions:

$$
\begin{aligned}
& u=-1, \quad \text { at } y=h_{1}(x), \\
& u=-1, \quad \text { at } y=h_{2}(x),
\end{aligned}
$$

where

$$
\begin{gathered}
h_{1}(x)=1+a \cos (2 \pi x), \\
h_{1}(x)=-d-b \cos (2 \pi x+\phi) .
\end{gathered}
$$


Moreover, $\mu(y)$ is the viscosity function. In [22], the authors considered the following viscosity variation in the dimensionless form:

$$
\mu(y)=e^{-\alpha y}
$$

This expression for the viscosity function has been also considered by many authors [17-21]. This assumption may be reasonable for physiological systems as reported in [19] that the viscosity of the gastric mucus (near the wall) varies as 1$10^{2} \mathrm{cP}$ while the viscosity of the chyme varies as $10^{3}-10^{6} \mathrm{cP}$. In order to use the regular perturbation method to solve (6), Afsar Khan et al. [22] have implemented the following approximate expression for the viscosity function $\mu(y)$ :

$$
\mu(y) \approx 1-\alpha y+\frac{\alpha^{2}}{2} y^{2}, \quad \text { for } \alpha \ll 1,
$$

where $\alpha$ has been used as a perturbation parameter.

\section{Notes on the Previous Results}

In [22], the authors mentioned that (6) is a nonlinear differential equation so that it is not possible to obtain a closed form solution; hence, a perturbation solution for $u$ has been obtained by expanding $u$ in the form

$$
u(x, y)=u_{0}(x, y)+\alpha u_{1}(x, y)+\alpha^{2} u_{2}(x, y)+\cdots .
$$

Unfortunately, the statement just mentioned above about the nonlinearity of the differential equation (6) is not correct, where (6) contains no nonlinear terms in the unknown function $u(x, y)$ or even any products for $u(x, y)$ with its derivatives. Accordingly, (6) is a linear differential equation and its exact solution is available; this is the subject of the next section. Such exact solution will be used to validate the accuracy of the numerical results obtained by Afsar Khan et al. [22] for the physical problem describing the peristaltic transport of a Jeffrey fluid with variable viscosity through a porous medium in an asymmetric channel. It is also important to refer to the fact that the exact solution is valid for any real value for the viscosity parameter $\alpha$. This means that the restriction $\alpha \ll 1$ made by the authors [22] can be avoided in the current analysis. Therefore, the present analytical solution may be considered as optimal for the current physical model.

\section{The Exact Solution}

On using the complete definition for $\mu(y)$ in (9), we can rewrite (6) in the following form, without any restriction on $\alpha$

$$
\frac{d p}{d x}=\frac{1}{\epsilon\left(1+\lambda_{1}\right)} \frac{\partial}{\partial y}\left[e^{-\alpha y} \frac{\partial u}{\partial y}\right]-\frac{1}{\mathrm{Da}} e^{-\alpha y}(u+1) .
$$

Equation (12) can be further simplified as

$$
\frac{\partial^{2} u}{\partial y^{2}}-\alpha \frac{\partial u}{\partial y}-\frac{\epsilon\left(1+\lambda_{1}\right)}{\mathrm{Da}}(u+1)=\epsilon\left(1+\lambda_{1}\right) \frac{d p}{d x} e^{\alpha y}
$$

Assuming that

$$
\Omega=\frac{\epsilon\left(1+\lambda_{1}\right)}{\mathrm{Da}}
$$

we have

$$
\frac{\partial^{2} u}{\partial y^{2}}-\alpha \frac{\partial u}{\partial y}-\Omega u=\Omega\left[1+\mathrm{Da} \frac{d p}{d x} e^{\alpha y}\right]
$$

which is a second-order linear partial differential equation. The exact solution of (15) is given as

$$
u(x, y)=-1-\mathrm{Da}\left(\frac{d p}{d x}\right) e^{\alpha y}+f_{1}(x) e^{\sigma_{1} y}+f_{2}(x) e^{\sigma_{2} y},
$$

where $f_{1}(x)$ and $f_{2}(x)$ are unknown functions. Further, $\sigma_{1}$ and $\sigma_{2}$ are given by

$$
\sigma_{1}=\frac{1}{2}\left(\alpha-\sqrt{\alpha^{2}+4 \Omega}\right), \quad \sigma_{2}=\frac{1}{2}\left(\alpha+\sqrt{\alpha^{2}+4 \Omega}\right) .
$$

Applying the boundary conditions (7), we obtain $f_{1}(x)$ and $f_{2}(x)$ as

$$
\begin{aligned}
& f_{1}(x)=\mathrm{Da}\left(\frac{d p}{d x}\right)\left[\frac{e^{\alpha h_{2}+\sigma_{2} h_{1}}-e^{\alpha h_{1}+\sigma_{2} h_{2}}}{e^{\sigma_{1} h_{2}+\sigma_{2} h_{1}}-e^{\sigma_{1} h_{1}+\sigma_{2} h_{2}}}\right], \\
& f_{2}(x)=\mathrm{Da}\left(\frac{d p}{d x}\right)\left[\frac{e^{\alpha h_{1}+\sigma_{1} h_{2}}-e^{\alpha h_{2}+\sigma_{1} h_{1}}}{e^{\sigma_{1} h_{2}+\sigma_{2} h_{1}}-e^{\sigma_{1} h_{1}+\sigma_{2} h_{2}}}\right] .
\end{aligned}
$$

In view of (16) and (18), we obtain the exact solution of (6) under the boundary conditions (7) as

$$
\begin{aligned}
u(x, y)= & -1+\operatorname{Da}\left(\frac{d p}{d x}\right) \\
& \times\left(\left[\frac{e^{\alpha h_{2}+\sigma_{2} h_{1}}-e^{\alpha h_{1}+\sigma_{2} h_{2}}}{e^{\sigma_{1} h_{2}+\sigma_{2} h_{1}}-e^{\sigma_{1} h_{1}+\sigma_{2} h_{2}}}\right] e^{\sigma_{1} y}\right. \\
& \left.+\left[\frac{e^{\alpha h_{1}+\sigma_{1} h_{2}}-e^{\alpha h_{2}+\sigma_{1} h_{1}}}{e^{\sigma_{1} h_{2}+\sigma_{2} h_{1}}-e^{\sigma_{1} h_{1}+\sigma_{2} h_{2}}}\right] e^{\sigma_{2} y}-e^{\alpha y}\right) .
\end{aligned}
$$

This exact solution can easily be verified by direct substitutions in (6) and the boundary conditions (7). Following Afsar Khan et al. [22], the volume flow rate in the wave frame is given by

$$
q=\int_{h_{2}}^{h_{1}} u d y .
$$

On using (16), we obtain from (20) that

$$
\begin{aligned}
q= & h_{2}(x)-h_{1}(x)-\frac{\mathrm{Da}}{\alpha}\left(\frac{d p}{d x}\right)\left(e^{\alpha h_{1}}-e^{\alpha h_{2}}\right) \\
& +\frac{f_{1}(x)}{\sigma_{1}}\left(e^{\sigma_{1} h_{1}}-e^{\sigma_{1} h_{2}}\right)+\frac{f_{2}(x)}{\sigma_{2}}\left(e^{\sigma_{2} h_{1}}-e^{\sigma_{2} h_{2}}\right) .
\end{aligned}
$$




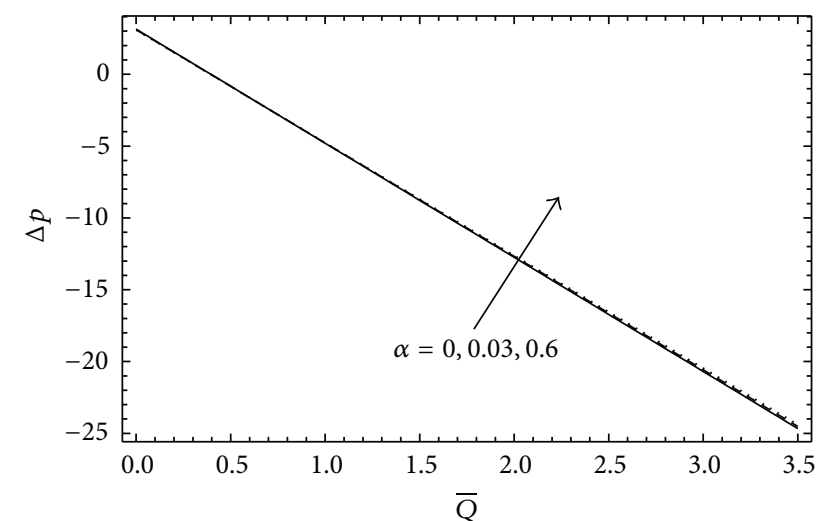

FIGURE 1: The pressure rise versus flow rate when $a=0.2 ; b=0.6 ; d=$ $0.8 ; \epsilon=0.3 ; \lambda_{1}=0.8 ; \mathrm{Da}=0.6 ; \phi=\pi / 4$.

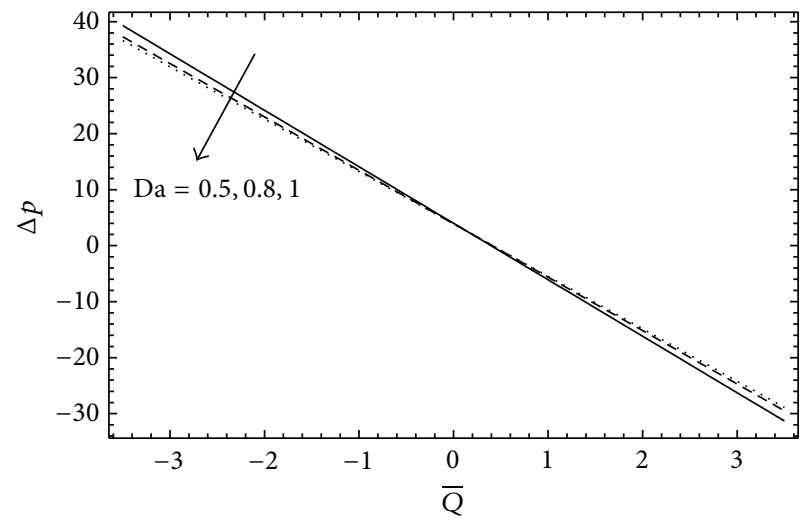

Figure 2: The pressure rise versus flow rate when $\alpha=0.01 ; a=0.2$; $b=0.6 ; d=0.8 ; \epsilon=0.3 ; \lambda_{1}=0.4 ; \phi=\pi / 4$.

Assume that

$$
\begin{aligned}
& \Omega_{1}(x)=\mathrm{Da}\left[\frac{e^{\alpha h_{2}+\sigma_{2} h_{1}}-e^{\alpha h_{1}+\sigma_{2} h_{2}}}{e^{\sigma_{1} h_{2}+\sigma_{2} h_{1}}-e^{\sigma_{1} h_{1}+\sigma_{2} h_{2}}}\right], \\
& \Omega_{2}(x)=\mathrm{Da}\left[\frac{e^{\alpha h_{1}+\sigma_{1} h_{2}}-e^{\alpha h_{2}+\sigma_{1} h_{1}}}{e^{\sigma_{1} h_{2}+\sigma_{2} h_{1}}-e^{\sigma_{1} h_{1}+\sigma_{2} h_{2}}}\right] .
\end{aligned}
$$

Therefore, the exact expression for the pressure gradient is given as

$$
\begin{aligned}
\frac{d p}{d x}=\left(\bar{Q}+h_{1}(x)-h_{2}(x)-(1+d)\right) \\
\times\left(\frac{\Omega_{1}(x)}{\sigma_{1}}\left(e^{\sigma_{1} h_{1}}-e^{\sigma_{1} h_{2}}\right)+\frac{\Omega_{2}(x)}{\sigma_{2}}\left(e^{\sigma_{2} h_{1}}-e^{\sigma_{2} h_{2}}\right)\right. \\
\left.\quad-\frac{\mathrm{Da}}{\alpha}\left(e^{\alpha h_{1}}-e^{\alpha h_{2}}\right)\right)^{-1},
\end{aligned}
$$

where $\bar{Q}$ is the average flux over one period and given by [22]

$$
\bar{Q}=q+1+d \text {. }
$$

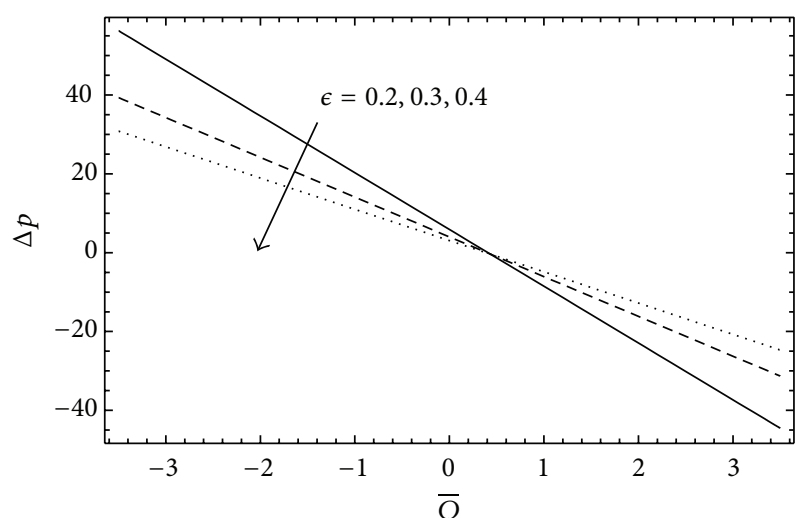

FIGURE 3: The pressure rise versus flow rate when $\alpha=0.01 ; a=0.2$; $b=0.6 ; d=0.8 ; \lambda_{1}=0.4 ; \mathrm{Da}=0.5 ; \phi=\pi / 4$.

The pressure gradient can be written in terms of the average flux as

$$
\frac{d p}{d x}=I_{1}(x) \bar{Q}+I_{2}(x),
$$

where

$$
\begin{aligned}
& I_{1}(x)=(1) \times\left(\frac{\Omega_{1}(x)}{\sigma_{1}}\left(e^{\sigma_{1} h_{1}}-e^{\sigma_{1} h_{2}}\right)\right. \\
& +\frac{\Omega_{2}(x)}{\sigma_{2}}\left(e^{\sigma_{2} h_{1}}-e^{\sigma_{2} h_{2}}\right) \\
& \left.-\frac{\mathrm{Da}}{\alpha}\left(e^{\alpha h_{1}}-e^{\alpha h_{2}}\right)\right)^{-1} \\
& I_{2}(x)=\left(h_{1}(x)-h_{2}(x)-(1+d)\right) \\
& \times\left(\frac{\Omega_{1}(x)}{\sigma_{1}}\left(e^{\sigma_{1} h_{1}}-e^{\sigma_{1} h_{2}}\right)+\frac{\Omega_{2}(x)}{\sigma_{2}}\left(e^{\sigma_{2} h_{1}}-e^{\sigma_{2} h_{2}}\right)\right. \\
& \left.-\frac{\mathrm{Da}}{\alpha}\left(e^{\alpha h_{1}}-e^{\alpha h_{2}}\right)\right)^{-1} .
\end{aligned}
$$

The dimensionless pressure rise is given exactly by

$$
\Delta P=\int_{0}^{1}\left(\frac{d p}{d x}\right) d x
$$

On inserting (25) into (27) we get

$$
\Delta P=\bar{Q} \int_{0}^{1} I_{1}(x) d x+\int_{0}^{1} I_{2}(x) d x .
$$

\section{Numerical Results}

In the previous section, the exact solution for the axial velocity has been obtained. Consequently, exact analytical expressions have been obtained for the pressure gradient and the pressure rise. The availability of such exact solutions is of great importance, especially in validating other approximate results, and they certainly would lead to better understanding 


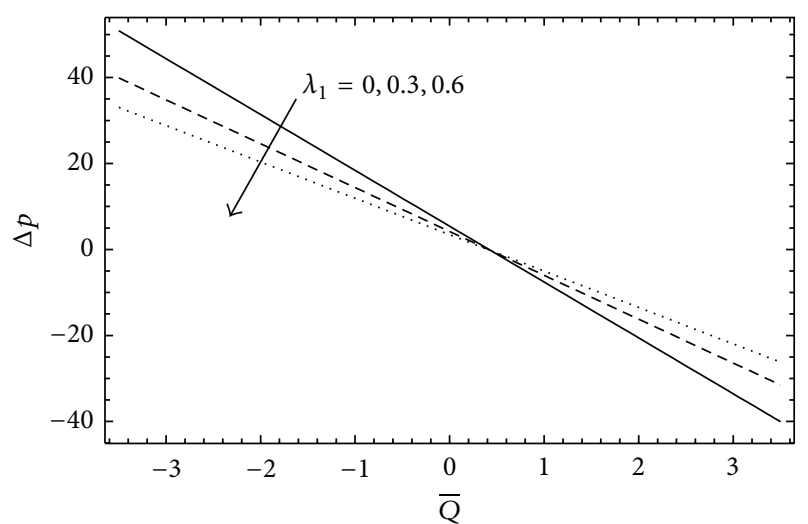

FIgURE 4: The pressure rise versus flow rate when $\alpha=0.01 ; a=0.2$; $b=0.6 ; d=0.8 ; \epsilon=0.3 ; \mathrm{Da}=0.8 ; \phi=\pi / 4$.

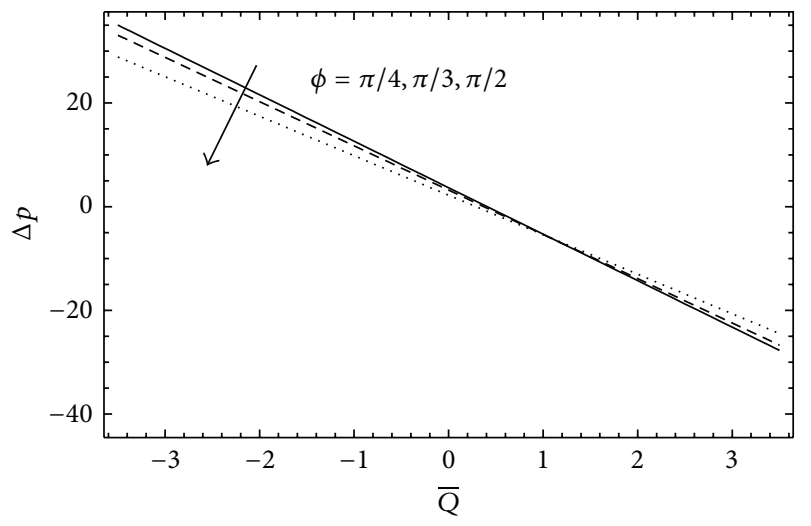

FIgURE 5: The pressure rise versus flow rate when $\alpha=0.01 ; a=0.2$; $b=0.6 ; d=0.8 ; \epsilon=0.3 ; \mathrm{Da}=0.8 ; \lambda_{1}=0.5$.

of the physical aspects of the model. Here, the obtained exact expressions are invested not only to explore the actual effects of various parameters on the velocity profiles and the pressure rise but also to validate the approximate results obtained in [22] by using the regular perturbation method. The relation between pressure rise and flow rate given by (28) is plotted in Figures 1-7 at the same numerical values taken by Afsar Khan et al. [22]. Figures 1-7 exhibit a linear relation between the variation of time-mean flow rate $\bar{Q}$ and the pressure rise $\Delta P$. Figure 1 shows the variation of $\Delta P$ with flow rate $\bar{Q}$ for different values of $\alpha$. The figure shows that the relation between pressure rise and flow rate is not greatly affected by the variation of the viscosity parameter at $\alpha=0,0.03,0.06$. However, the situation was completely different regarding the results depicted in Figure 1 by Afsar Khan et al. [22]. Further, it is observed from the current Figure 1 that $-25 \leq \Delta P \leq 5$, while it was observed from Figure 1 in [22] that $-10 \leq \Delta P \leq$ 55.

Figure 2 represents the variation of $\Delta P$ with the flow rate $\bar{Q}$ for different values of $\mathrm{Da}$. The current results in Figure 2 reveal that slight decrease in $\triangle P$ occurs with increasing $\mathrm{Da}$ when $-3.5 \leq \bar{Q} \leq 0$; however, Figure 2 in [22] showed that $\Delta P$ increases with increasing $\mathrm{Da}$ when $\bar{Q} \leq 2.55$.

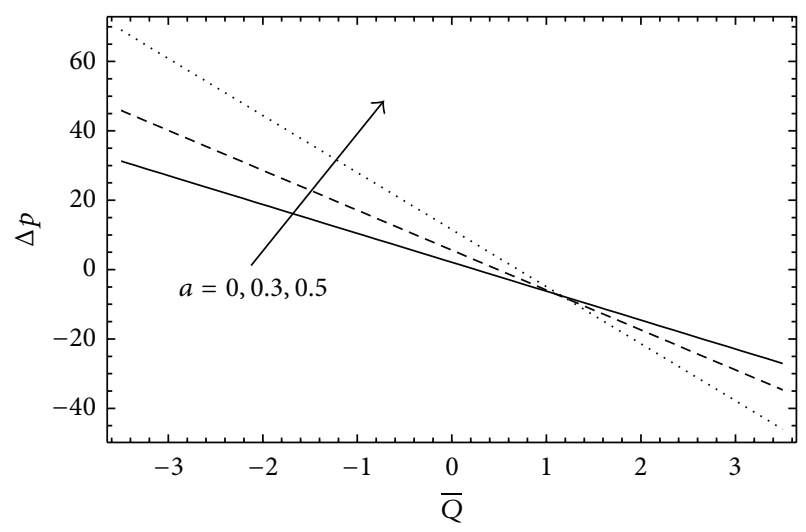

FIGURE 6: The pressure rise versus flow rate when $\alpha=0.01 ; b=0.6$; $d=0.8 ; \epsilon=0.3 ; \lambda_{1}=0.4 ; \mathrm{Da}=0.5 ; \phi=\pi / 4$.

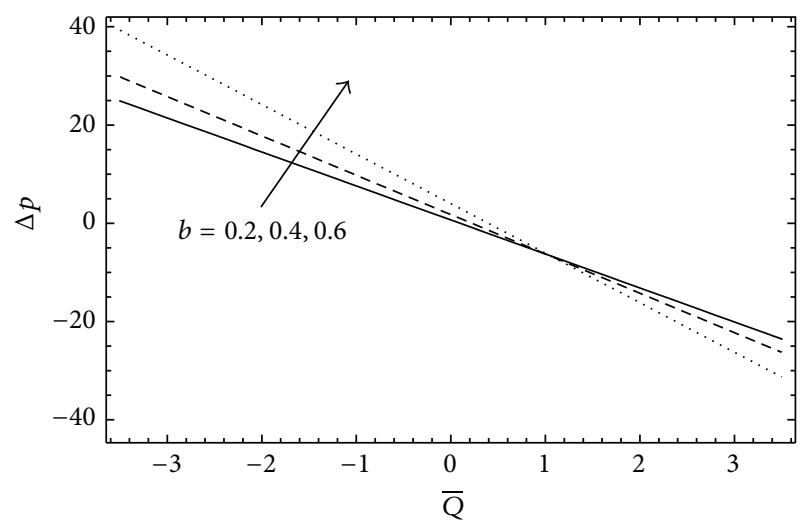

FIgURE 7: The pressure rise versus flow rate when $\alpha=0.01 ; a=0.2$; $d=0.8 ; \epsilon=0.3 ; \lambda_{1}=0.4 ; \mathrm{Da}=0.5 ; \phi=\pi / 4$.

This comparison shows different behaviour for the relation between pressure rise and flow rate when studying the effect of Da. Figures 3 and 4 represent the graphs of pressure rise $\Delta P$ with the flow rate $\bar{Q}$ for different values of $\epsilon$ and $\lambda_{1}$. It is observed that the pumping rate decreases with increase of $\epsilon$ and $\lambda_{1}$. Although the same behaviour has been obtained by Afsar Khan et al. [22] in Figures 3 and 4 for the relation between pressure rise and flow rate when studying the effects of $\epsilon$ and $\lambda_{1}$, the ranges of $\bar{Q}$ at which the pumping rate decreases with increase of $\epsilon$ and $\lambda_{1}$ differ from those observed in Figures 3 and 4 in [22]. Figure 5 indicates the variation of $\Delta P$ versus $\bar{Q}$ for different values of phase difference $\phi$. It is clarified in Figure 5 that the pumping rate decreases with the increase of $\phi$ and this behaviour agrees with the corresponding results introduced by Figure 5 in [22] but at different range for the flow rate $\bar{Q}$. The exact influences of the waves amplitudes $a$ and $b$ on the variation of $\Delta P$ versus $\bar{Q}$ are plotted in Figures 6 and 7, respectively. Although the current exact influences for $a$ and $b$ are similar to the approximate results depicted by Figures 6 and 7 in [22], they also occur at different range for $\bar{Q}$.

Regarding the exact effects of $\alpha, q, \phi$, and Da on the axial velocity profiles, we have plotted such effects in Figures 8, 9, 


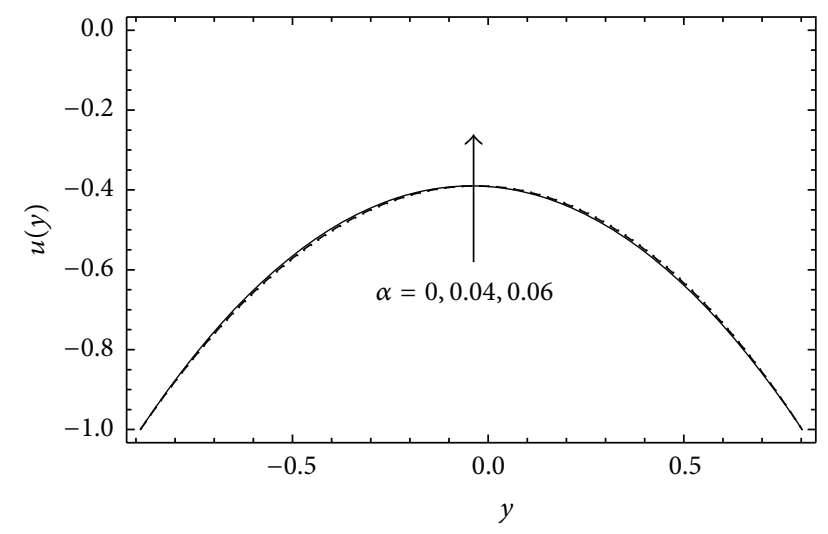

FIGURE 8: Axial velocity versus $y$ at $a=0.2 ; b=0.6 ; d=0.8 ; \epsilon=0.2$; $\lambda_{1}=1 ; \mathrm{Da}=1 ; x=\pi / 6 ; q=-1 ; \phi=\pi / 2$.

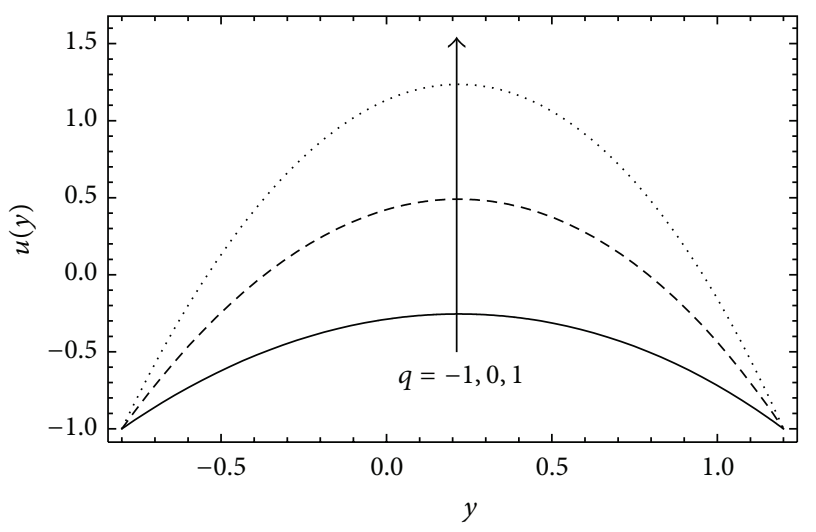

FIgURE 9: Axial velocity versus $y$ at $\alpha=0.05 ; a=0.2 ; b=0.6 ; d=0.8$; $\epsilon=0.2 ; \lambda_{1}=1 ; \mathrm{Da}=1 ; x=0 ; \phi=\pi / 2$.

10,11 , and 12 . Figures 8 and 11 declare that the axial velocity is not affected by the variations of the viscosity parameter when $\alpha=0,0.04,0.06$ and Darcy's number at $\mathrm{Da}=0.5,1$, 1.2. Indeed, these exact effects of $\alpha$ and $\mathrm{Da}$ on $u(y)$ differ from those approximately obtained and depicted in Figures 8 and 11 in [22]. Here, it may be important to mention that $u(y)$ should be affected by the variations of $\alpha$ and $\mathrm{Da}$, but in other ranges than those taken in [22]; note that the very small values of $\alpha$ considered by Afsar Khan et al. [22] were chosen in such way to meet the requirements of the perturbation method. However, there are not any restrictions on $\alpha$ in the current analysis. The most important notice here is that the velocity curves presented in Figure 8 by Afsar Khan et al. [22] do not satisfy the boundary conditions. Such drawbacks in the results can be also observed in Figures 10, 11, and 12 in [22]. Figure 12 of the current study shows that the axial velocity is influenced by the variation in $\mathrm{Da}$ at smaller values for $\mathrm{Da}$ than those considered by Afsar Khan et al. [22]. Besides, the current exact curves for the axial velocity satisfy the boundary conditions as shown in Figures 8-12.

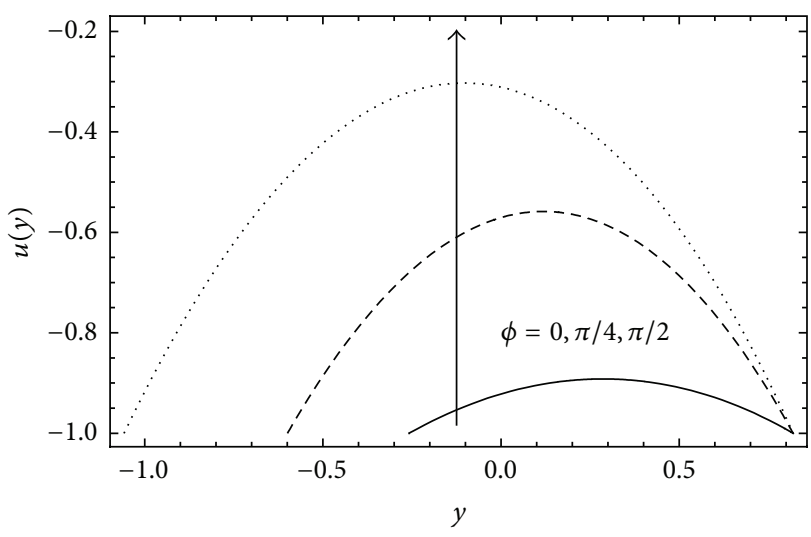

FIgURE 10: Axial velocity versus $y$ at $\alpha=0.05 ; a=0.2 ; b=0.6 ; d=$ $0.8 ; \epsilon=0.2 ; \lambda_{1}=1 ; \mathrm{Da}=1 ; x=\pi / 2 ; q=-1$.

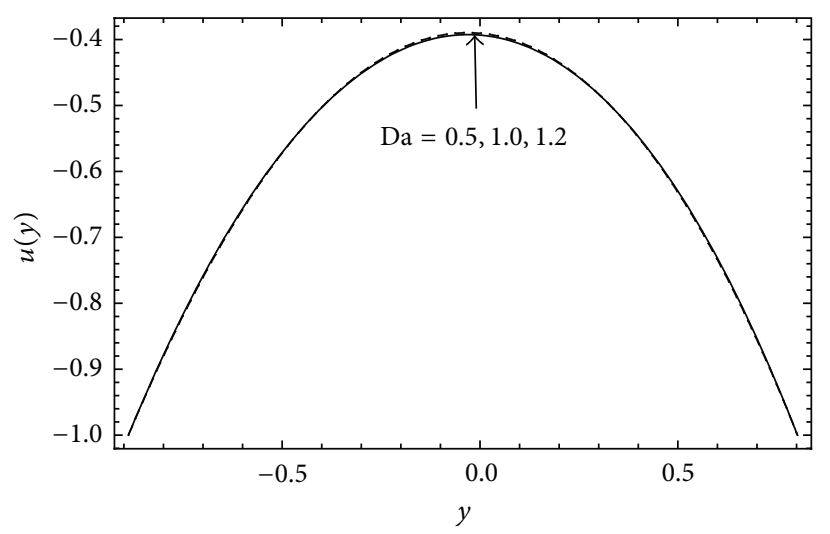

FIgURE 11: Axial velocity versus $y$ at $\alpha=0.05 ; a=0.2 ; b=0.6 ; d=0.8$; $\epsilon=0.2 ; \lambda_{1}=1 ; q=-1 ; x=\pi / 6 ; \phi=\pi / 2$.

\section{Conclusion}

In this paper the physical model describing the influence of viscosity variation on peristaltic flow in an asymmetric channel has been reanalyzed in view of new exact solutions. The main advantage of these exact solutions is the avoidance of any kind of restrictions on the viscosity parameter, unlike the study [22] in which a restriction has been put on $\alpha, \alpha \ll$ 1 , to achieve the requirements of the regular perturbation method. The obtained exact solutions have been used to study the effects of the viscosity parameter, Daray's number, porosity, amplitude ratio, Jeffrey fluid parameter, and the amplitudes of the waves on the pressure rise and the axial velocity. The obtained exact results have been compared with other approximate analytical results obtained in the literatures by using the regular perturbation method [22]. The comparisons clarified that there are remarkable differences between the current exact results and those approximately obtained in the literatures. The inaccurate numerical results derived in [22] come back to the convergence issue which was not addressed by the authors. A final note on the current comparative study is that when it is difficult to achieve the exact solutions of the considered physical problem we instead 


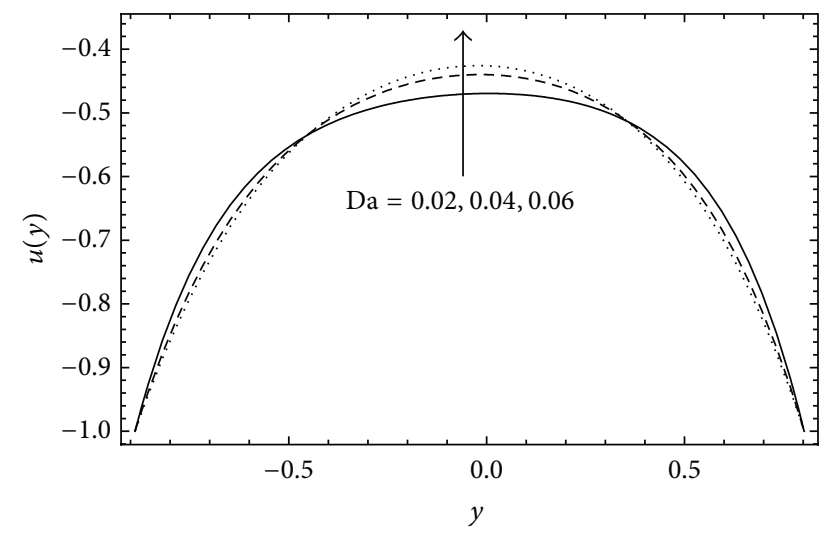

Figure 12: Axial velocity versus $y$ at $\alpha=0.05 ; a=0.2 ; b=0.6 ; d=$ $0.8 ; \epsilon=0.2 ; \lambda_{1}=1 ; q=-1 ; x=\pi / 6 ; \phi=\pi / 2$.

search for the approximate solutions taking into account the convergence of such solutions as pointed out in $[23,24]$.

\section{Conflict of Interests}

The authors declare that there is no conflict of interests regarding the publication of this paper.

\section{References}

[1] T. W. Latham, Fluid motion in a peristaltic pump [M.S. thesis], MIT, Cambridge, Mass, USA, 1966.

[2] A. H. Shapiro, M. Y. Jaffrin, and S. L. Weinberg, "Peristaltic pumping with long wavelengths at low Reynolds number," Journal of Fluid Mechanics, vol. 37, no. 4, pp. 799-825, 1969.

[3] T.-F. Zien and S. A. Ostrach, "A long wave approximation to peristaltic motion," Journal of Biomechanics, vol. 3, no. 1, pp. 63$75,1970$.

[4] J.-S. Lee and Y.-C. Fung, "Flow in nonuniform small blood vessels," Microvascular Research, vol. 3, no. 3, pp. 272-287, 1971.

[5] L. M. Srivastava, V. P. Srivastava, and S. N. Sinha, "Peristaltic transport of a physiological fluid: part I-flow in non-uniform geometry," Biorheology, vol. 20, no. 2, pp. 153-166, 1983.

[6] S. Takabatake, K. Ayukawa, and A. Mori, "Peristaltic pumping in circular cylindrical tubes: a numerical study of fluid transport and its efficiency," Journal of Fluid Mechanics, vol. 193, pp. 267$283,1988$.

[7] L. M. Srivastava and V. P. Srivastava, "Peristaltic transport of a power-law fluid: application to the ductus efferentes of the reproductive tract," Rheologica Acta, vol. 27, no. 4, pp. 428-433, 1988.

[8] D. Tang and M. C. Shen, "Nonstationary peristaltic transport of a heat-conducting fluid," Journal of Mathematical Analysis and Applications, vol. 174, no. 1, pp. 265-289, 1993.

[9] J. C. Misra and S. K. Pandey, "Peristaltic transport of a particle-fluid suspension in a cylindrical tube," Computers \& Mathematics with Applications, vol. 28, no. 4, pp. 131-145, 1994.

[10] J. C. Misra and S. K. Pandey, "Peristaltic transport in a tapered tube," Mathematical and Computer Modelling, vol. 22, no. 8, pp. 137-151, 1995.

[11] V. P. Srivastava and L. M. Srivastava, "Influence of wall elasticity and Poiseuille flow on peristaltic induced flow of a particle-fluid mixture," International Journal of Engineering Science, vol. 35, no. 15 , pp. 1359-1386, 1997.

[12] O. Eytan, A. J. Jaffa, and D. Elad, "Peristaltic flow in a tapered channel: Application to embryo transport within the uterine cavity," Medical Engineering \& Physics, vol. 23, no. 7, pp. 473482, 2001.

[13] K. Vajravelu, S. Sreenadh, and V. R. Babu, "Peristaltic transport of a Herschel-Bulkley fluid in an inclined tube," International Journal of Non-Linear Mechanics, vol. 40, no. 1, pp. 83-90, 2005.

[14] K. S. Mekheimer and Y. Abd elmaboud, "Peristaltic flow through a porous medium in an annulus: application of an endoscope," Applied Mathematics \& Information Sciences, vol. 2, no. 1, p. 103, 2008.

[15] K. S. Mekheimer and Y. Abd elmaboud, "Peristaltic flow of a couple stress fluid in an annulus: application of an endoscope," Physica A, vol. 387, no. 11, pp. 2403-2415, 2008.

[16] K. de Vries, E. A. Lyons, G. Ballard, C. S. Levi, and D. J. Lindsay, "Contractions of the inner third of the myometrium," American Journal of Obstetrics and Gynecology, vol. 162, no. 3, pp. 679-682, 1990.

[17] A. El Hakeem, A. E. M. El Misiery, and I. I. El Shamy, "Hydromagnetic flow of fluid with variable viscosity in a uniform tube with peristalsis," Journal of Physics A, vol. 36, no. 31, pp. 85358547, 2003.

[18] N. T. Eldabe, E. M. Elghazy, and A. Ebaid, "Closed form solution to a second order boundary value problem and its application in fluid mechanics," Physics Letters A, vol. 363, no. 4, pp. 257-259, 2007.

[19] A. Ebaid, "A new numerical solution for the MHD peristaltic flow of a bio-fluid with variable viscosity in a circular cylindrical tube via Adomian decomposition method," Physics Letters A, vol. 372, no. 32, pp. 5321-5328, 2008.

[20] S. Nadeem and N. S. Akbar, "Peristaltic flow of a Jeffrey fluid with variable viscosity in an asymmetric channel," Zeitschrift fur Naturforschung A, vol. 64, no. 11, pp. 713-722, 2009.

[21] S. Nadeem and N. S. Akbar, "Effects of heat transfer on the peristaltic transport of MHD Newtonian fluid with variable viscosity: application of Adomian decomposition method," Communications in Nonlinear Science and Numerical Simulation, vol. 14, no. 11, pp. 3844-3855, 2009.

[22] A. Afsar Khan, R. Ellahi, and K. Vafai, "Peristaltic transport of a Jeffrey fluid with variable viscosity through a porous medium in an asymmetric channel," Advances in Mathematical Physics, vol. 2012, Article ID 169642, 15 pages, 2012.

[23] A. Ebaid, "Approximate analytical solution of a nonlinear boundary value problem and its application in fluid mechanics," Zeitschrift fur Naturforschung A, vol. 66, no. 6-7, pp. 423-426, 2011.

[24] A. Ebaid and E. H. Aly, "Exact analytical solution of the peristaltic nanofluids flow in an asymmetric channel with flexible walls and slip condition: application to the cancer treatment," Computational and Mathematical Methods in Medicine, vol. 2013, Article ID 825376, 8 pages, 2013. 


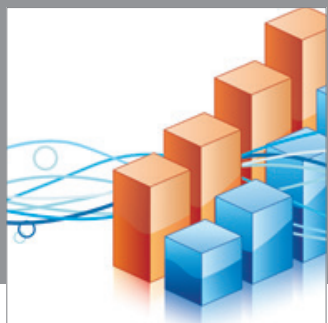

Advances in

Operations Research

mansans

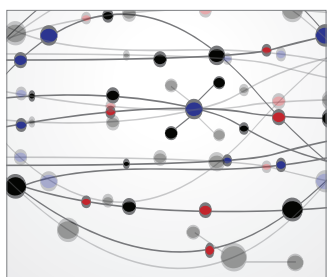

The Scientific World Journal
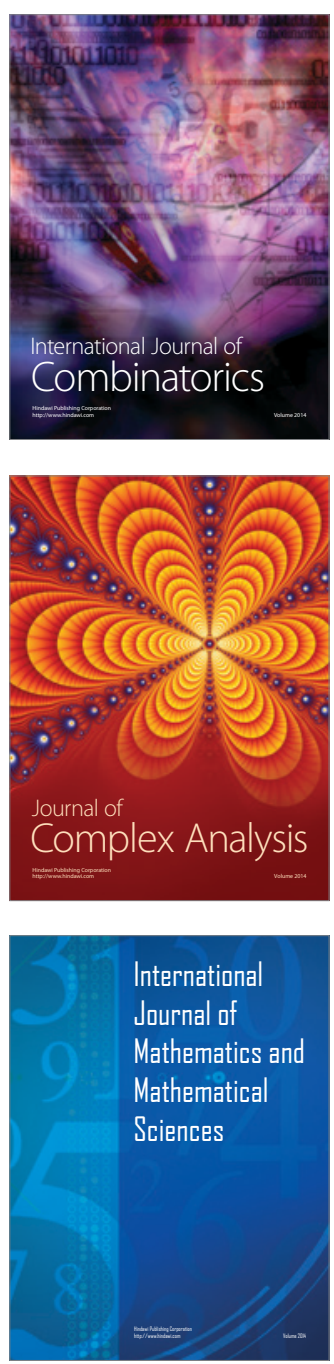
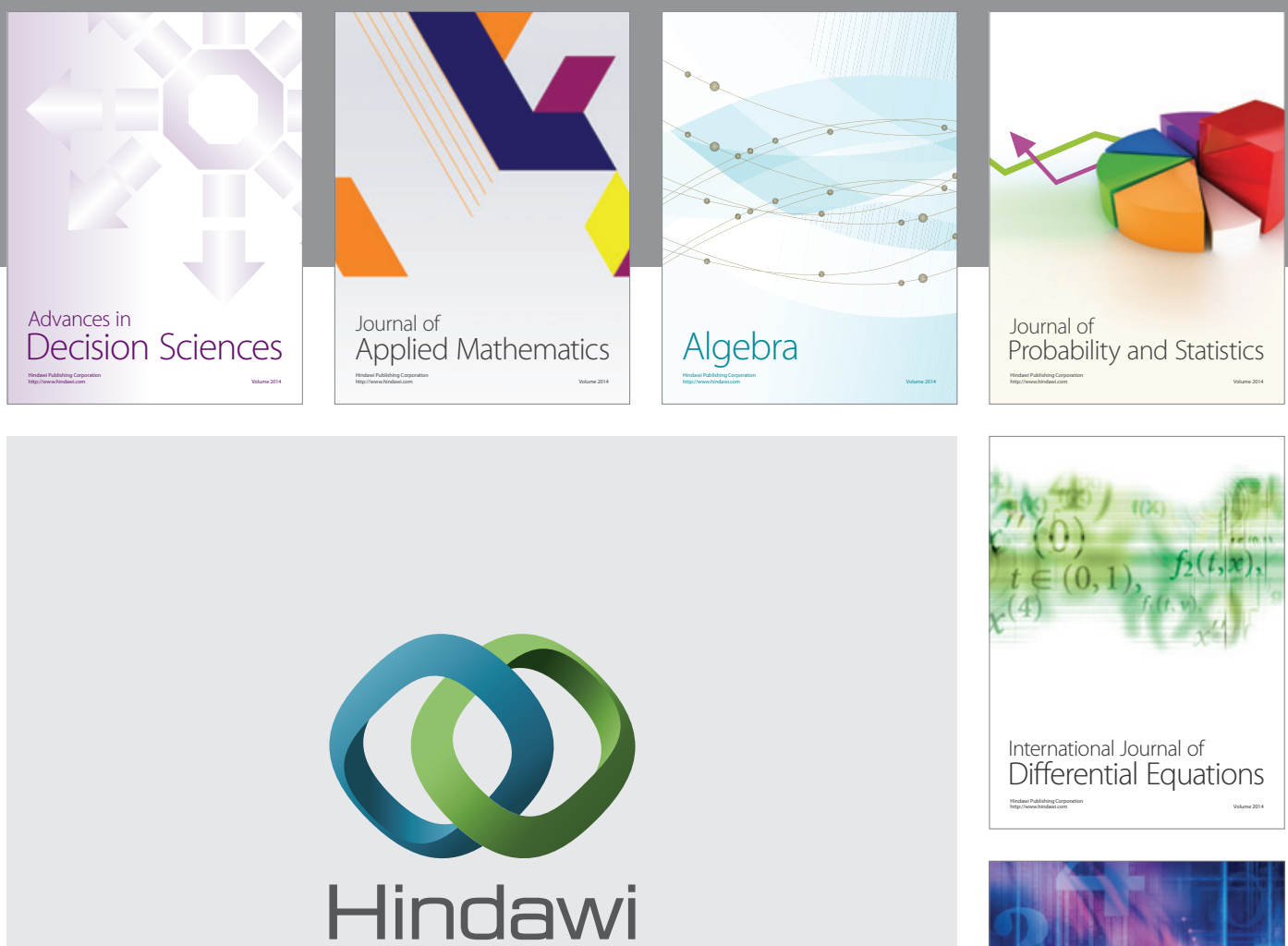

Submit your manuscripts at http://www.hindawi.com
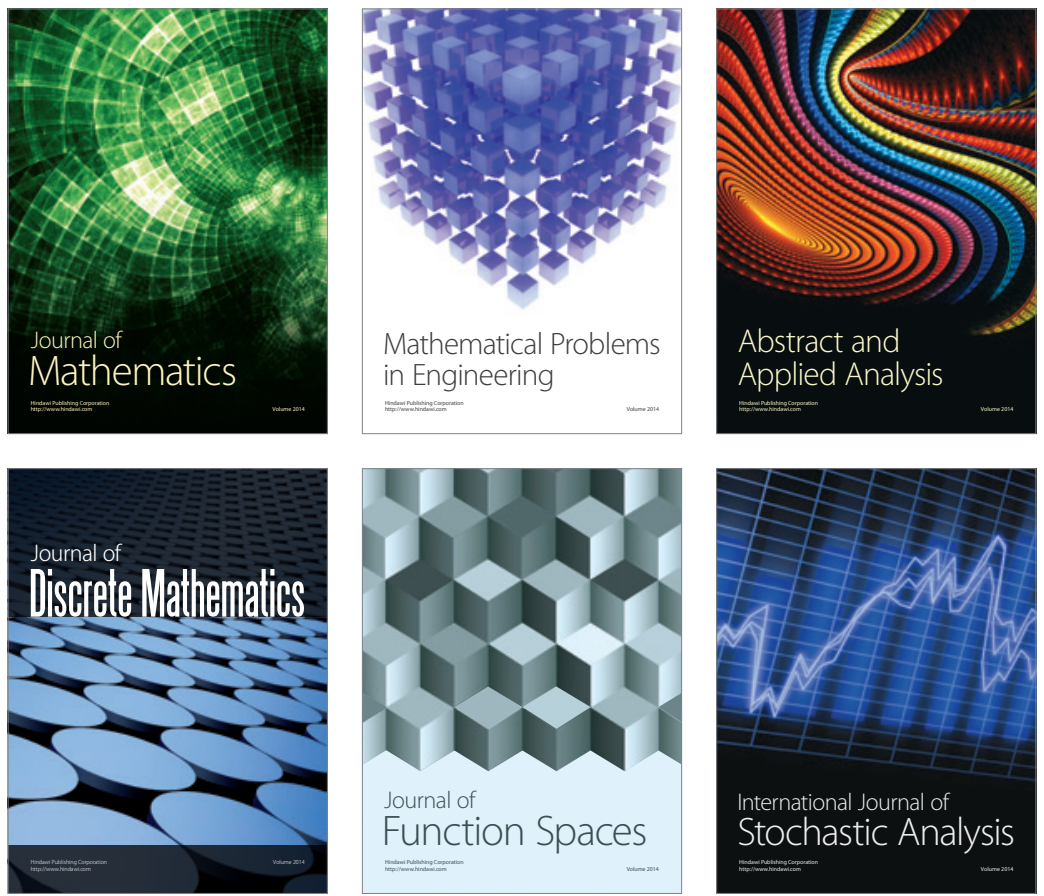

Journal of

Function Spaces

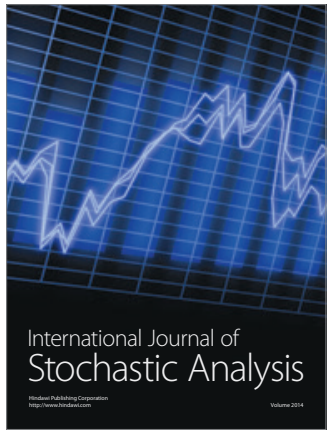

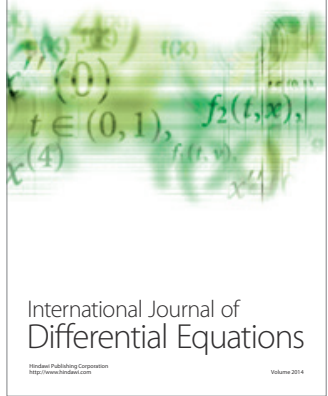
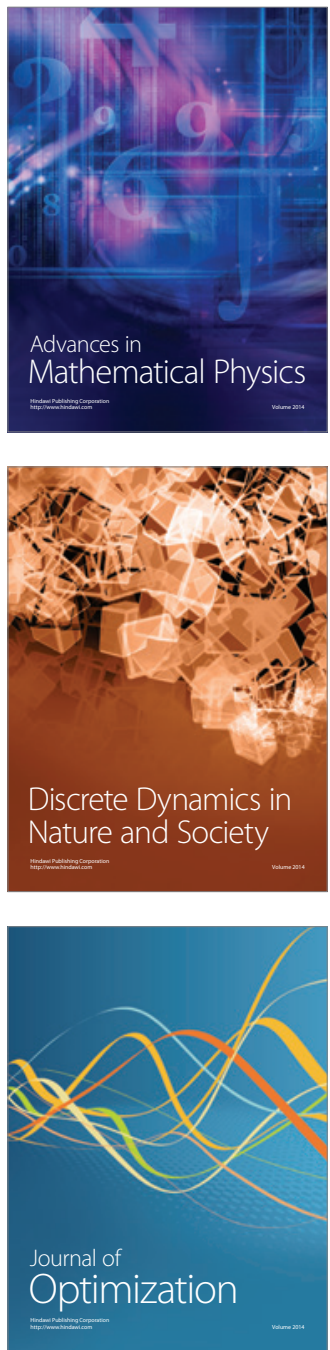